\title{
Infección por virus varicela zoster en enfermedad de Hodgkin del niño en estadios avanzados III y IV
}

\author{
M.C. Paola Zolezzi R. ${ }^{1,2}$; M.C. Maria Soledad Wenzel A. ${ }^{1}$; \\ E.U. María Olga Delgado S. ${ }^{3}$ \\ Varicella-zoster infection in children with advanced \\ Hodgkin's disease
}

Over a 10-year period, among 22 children with Hodgkin's disease (stages III and IV), 10 (45.5\%) developed varicella-zoster virus (VZV) infection, varicella in 8 cases $(36.4 \%$ ) and herpes zoster $(\mathrm{HZ})$ in $3(13.6 \%)$ (one patient had raricella and six months later). Three patients with varicella had significant pneumonitis, one of them showed clinical evidence of dissemination and died. Two patients had localized $\mathrm{HZ}$ and one had disseminated $\mathrm{HZ}$ without visceral involvement. All cases of VZV infections ocurred in the first year of treatment: the primary infection presented while patients were under induction therapy and the secondary one after radiotherapy.

(Key wonds: Hodgkin's disease, immunosupression, varicella zoster virus, infection, chjckenpox, herpes zoster.)

1. Instituto de Pediatría, Facultad de Medicina, Universidad Austral de Chile.

2. Instituto de Hematologia, Facultad de Medicina, Universidad Austral de Chile.

3. Servicio de Pediatria, Hospital Regional de Valdivia.
El virus varicela zoster (VVZ) representa una amenaza para el paciente inmunocomprometido en tratamiento quimioterápico, tanto la infección primatia (varicela) como su reactivación (herpes zoster) $(\mathrm{HZ})^{1}$. Constituye la infección más co- 
mún en la enfermedad de Hodgkin $(E H)^{2}$, con aumento de su frecuencia en los últimos aros, alcanzando cifras entre $25 \%{ }^{3}$ y $35 \%{ }^{4}$, que aumentan a $56 \%{ }^{4}$ cuando se utilizan, en nifros con EH en estadios avanzados, tratamientos combinados de quimioterapia (QMT) y radioterapia (RDT), que, a su vez, producen, en etapas I?-B a IV, 60\% de sobrevida libre de recidiva a cinco años ${ }^{5}$. Alteraciones del sistema inmune ${ }^{6}$, asociadas o no a efectos de la QMT, RDT o ambas $^{3,4,7}$, no han logrado explicar totalmente el curso más prolongado que la infectión por VVZ suele presentar en estos pacientes, adquiriendo en ocasiones caracteres de diseminación, a veces de curso fulminante ${ }^{2,8-10}$. Tanto la profilaxis $^{11,12}$ como el mejor tratamiento de la infección por VVZ en los pacientes con cáncer son materia de revisiones recientes ${ }^{13,14}$.

La alta incidencia de infección por VVZ y sus complicaciones en un grupo de pacientes con EH, menores de 15 años, en estadios avanzados III y IV, sometidos a un tratamiento combinado de QMT y RDT, durante el decenio 1976-1985, en la $X$ Region, es el motivo de este análisis.

\section{PACIENTES Y METODO}

Se analizan 10 pacientes con infección por VVZ de un total de 22 menores de 15 años, en estadios III y IV de EH, diagnosticados por biopsia ganglionat compatible, clasificados histológicamente según Rye y etapificados segín Ann Arbor. Todos los pacientes fueron tratados con QMT: esquema de COPP modificado que incluyó ciclofosfamida, vincristina, procarbazima y prednisona, más RDT en dosis dependientes de la magnitud de la $\mathrm{EH}^{15}$.

El diagnóstico de varicela se hizo en base a la presentación de lesiones papulovesiculosas, generalizadas típicas, asociadas con un cuadro clínico compatible y la evolución esperada. Se definió neumonitis varicelatosa como la presencia de infiltrados nodulares de bordes difusos bilaterales en la $\mathbf{R x}$ de tórax durante el curso de la varicela. Se diagnosticó encefalitis en presencia de signos y síntomas de disfunción cerebral con pleiocitosis del $\mathrm{LCR}^{9}$.

El diagnóstico de H2 localizado se basó en la presencia de lesiones papulovesiculares típicas, distribuidas en uno a tres dermátomos sensitivos. El diagnóstico de HZ diseminado se basó en la presencia de más de cinco lesiones vesiculares fueta del dermátomo primario comprometido, acompañado o no de compromiso visce. $\mathrm{ral}^{4,10}$. No se hicieton estudios virológicos.

Se analizaron la incidencia y el tipo de infección por VVZ, sus complicaciones, el momento de su aparición en el transcurso de la $\mathrm{EH}$, las modificaciones que provoca en el tratamiento y su significado en eI posterior curso clinico de la EH.
Los pacientes han sido controladas por un período de 3 a 13 años.

\section{RESULTADOS}

En este grupo de 22 menores de 15 años con EH en estadios III y IV, tratados con QMT mas RDT en el decenio 1976-1985, 10 pacientes $(45,5 \%)$ presentaron infección por VVZ.

En 8 casos $(36,4 \%)$ se diagnosticó infección primaria, cuya evolución duró entre diez y quince días en cinco. En tres casos êsta se complicó con neumonitis varicelatosa, que se resolvió en dos semanas en uno; en el segundo se asoció con encefalitis de recuperación lenta $y$, en el tercero, se acompañó de diseminación progresiva y muerte a las tres semanas de iniciada la erupción. Estos tres pacientes correspondían a estadios IV.B de su EH.

Tres pacientes presentaron $\mathrm{HZ}(13,6 \%)$ de tipo localizado en dos casos y de tipo cutáneo diseminado sin compromiso visceral en el tercero. Este último había presentado una varicela común seis meses antes. Las lesiones herpéticas se resolvieron en treinta dias en los tres casos.

En relación al momento de aparición de la infección por VVZ, todos los casos de varicela se presentaron antes de transcurridos cuatro meses, desde el diagnóstico de la $\mathrm{EH}$, en dos durante la estadificación y en el resto durante la QMT de inducción. Los casos de $\mathrm{HZ}$ ocurrieron entre cuatro y diez meses después de comprobada la EH, habiendo recibido todos RDT y encontrándose en QMT de mantención. No se han observado nuevas infecciones por VVZ una vez finalizado el tratamiento de la $\mathrm{EH}$.

El tratamiento QMT de la EH se postergó entre 15 y 21 días en los tres casos de varicela clásica y en los tres de $\mathrm{HZ}$, por encontrarse en remisión completa de su EH. No se suspendió la QMT en los tres pacientes con varicela complicada.

El tratamiento de la infección por VVZ fue sintomático en nueve, y con aciclovir en el último paciente de la serie, cuya varicela era benigna.

En cuanto al significado de la infección por VVZ en el pronóstico a largo plazo de la $\mathbf{E H}$, exceptuando el paciente que falleció a los 21 dias como consecuencia de una diseminación varicelatosa, la muerte se produjo entre 4 y 18 meses después de la infección en cuatro pacientes: se trata de los otros dos casos que sufrieron neumonitis, un caso de $\mathrm{HZ}$ localizado y el paciente que 
tuvo waricela durante la estadificación y que seis meses después presentó $\mathrm{HZ}$ diseminado. En cambio, no se han registrado defunciones en los otros 12 pacientes del total de 22 con EH en estadios III y IV que no han presentado infecciones por $V V Z(p<0,01)$.

\section{DISCUSION}

En estos niños con EH en estadios Ill y IV, la frecuencia de infección por VVZ es levemente inferior a la del grupo de Stanford de $56 \%^{4}$. La enfermedad se presentó en los primeros meses siguientes al diagnóstico de la $\mathbf{E H}$, predominando la infección primaria, a diferencia de otras series en las cuales $80 \%$ de las infecciones sucedieron durante el primer año, después de finalizado el tratamiento, y mostraron, preferentemente, manifestaciones de tipo $\mathrm{HZ}^{4,10}$. La esplenecto. mía, que para algunos favorecería una mayor incidencia de infecciones por $\mathrm{VVZ}^{7}$, no habria jugado un rol en nuestro grupo, ya que sólo 2 de estos 10 pacientes fueron sometidos a lapa. rotomia de clasificación. Consideramos importante en la presentación de la infección primaria el papel desempeñado por la QMT, y en la pre. sentación del HZ la asocjación de QMT más RDT, ya que es conocido el compromiso inmunológico que ambas produce $n^{4,6}$. Atribuimos a la edad y a la alta ruralidad de nuestros pacientes la mayor incidencia de varicela. La neumonitis, complicación que se presentó en tres de nuestros casos, con diseminación fatal en uno, cuadro clínico descrito en otras series ${ }^{4,16}$, es responsable -asociada o no a encefalitis- del $7 \%$ de mortalidad registrada en los niños con cáncer y varice. $1 a^{9}$. Nuestra incidencia de $\mathrm{HZ}$ es inferior a la descrita por el grupo del Saint Jude, de $21,6 \%^{10}$, siendo también diferente el curso clínico, sin mayores complicaciones, observado en estos pacientes. Nuestro grupo adoptó el criterio de postergar el tratamiento en los casos estimados en remisión completa. Lamentablemente las formas más graves se presentaron en niños, en los cuales la recomendación de suspender el tratamiento durante un promedio de 21 dias, 17 constituye un riesgo, debido a la probable progresión de la enfermedad de base. La significación pronóstica que tiene la infección por VVZ en EH ha sido analizada por el grupo de Stan- ford ${ }^{4} y$, a diferencia de ellos, nosotros observamos en el seguimiento a largo plazo que la letalidad por EH, que en nuestras series es de $22,7 \%{ }^{15}$, está dada por pacientes provenientes del grupo que sufrieron infecciones por VVZ.

Para prevenir o modificar la infección prima. ria por VVZ en los nifios con cáncer se ha reco. mendado administrar, antes de 48 a 72 horas después de la exposición al virus, plasma inmune zoster, $7 \mathrm{ml} \mathrm{x} \mathrm{kg} \mathrm{peso} \mathrm{e.v.}{ }^{12}$ o inmunoglobulina varicela zoster, $125 \mathrm{U}$. i.m. por cada $10 \mathrm{~kg}$ de peso, máximo $625 \mathrm{U}^{18}{ }^{18}$. Trabajos controlados han demostrado la efectividad de la vacuna con VVZ vivo (vacuna Oka), cuyo uso ha sido aceptado recientemente en algunos países, debiendo recordarse que las personas inmunodeprimidas constituyen el grupo más expuesto a las complicaciones derivadas de la vacunación misma ${ }^{11,19}$. Dado que para el tratamiento de la varicela en los pacientes de alto riesgo se requiere una terapia antiviral eficaz, se ha utilizado interferon de leucocitos humanos, en dosis promedio de $4,2 \times$ $10^{4} \mathrm{U} . \times \mathrm{kg}$ peso, administrado diariamente hasta la desaparición de las lesiones, lo que ocurre alrededor de los 6 días, disminuyendo la severidad del cuadro clínico y su mortalidad ${ }^{20}$. Resul. tados similares se han obterido con otro agente antiviral, la vidarabina, usada también antes de las 72 horas de la exposición, en dosis de $10 \mathrm{mg}$ $x \mathrm{~kg}$ peso diario en infusión e.v. de 12 horas por cinco dias, en concentración que no exceda de $0,5 \mathrm{mg} / \mathrm{ml}$. Su toxicidad comparada con la del interferon es menor, debiendo precisarse aún su dosificación y el período de administración óptimo ${ }^{14}$. Ultimamente se ha generalizado el uso de aciclovir, dada su mayor eficacia en impedir el desarrollo de la neumonitis varicelatosa y por haberse demostrado neurotoxicidad mínima en comparación con la producida por la vidarabina $^{21-23}$, en dosis de $500 \mathrm{mg} \mathrm{x} \mathrm{m}^{2}$ e.v. cada 8 horas durante 7 dias ${ }^{1}, 19$. Para los pacientes inmunosuprimidos con $\mathrm{HZ}$ y mayor riesgo de diseminación cutánea o visceral se recomienda emplear aciclovir, que acelera la resolución de las lesiones o, en su defecto, vidarabina, ambas en las dosis respectivas ya mencionadas ${ }^{19}$. El último de nuestros pacientes recibió aciclovir, pues contamos con este medicamento desde 1985. Dada la gravedad de la infección por VVZ en estos pacientes, parece recomendable protegerlos precozmente, en caso de contacto, mediante inmunización pasiva o drogas antivirales eficaces. 


\section{RESUMEN}

De 22 menores de 15 años con enfermedad de Hodgkin (EH) en estadios III y IV, estudiados en el Hospital de Valdivia, en el periodo $1976-1985$, diez $(45,5 \%)$ presentaron infección por virus varicela zoster (VVZ), correspondiendo 8 casos $(36,4 \%)$ a varicela y $3(13,6 \%)$ a herpes zoster (HZ) (un caso presentó varicela y seis meses después $\mathrm{HZ}$ ). Tres casos de varicela se complicaron de neumonitis, con diseminación de la infección y muerte a los 21 días en uno de ellos. Dos de los casos de HZ fueron de tipo localizado y uno cutáneo diseminado sin com. promiso visceral. Todos los episodios se presentaron durante el primer año de tratamiento: la infección primaria mientras los pacientes recibían QMT de inducción y la reactivación (HZ) después de la RDT. No se han observado nuevas manifestaciones de infección por VVZ una vez finalizado el tratamiento de la EH. La severidad que la infección por VVZ adquiere en niños con EH avanzada obliga al uso precoz de inmunización pasiva o drogas antivirales.

(Palabras claves: enfermedad de Hodgkin, inmunosupresión, virus varicela zoster, varicela, herpes zoster.)

\section{REFERENCIAS}

1. Balfour H.H.: Intravenous acyclovir therapy for varicella in immunocompromised children. J Pediatr 1984; 104: 134-136.

2. Bonm $K$.: Increasing frequency of acute myeloid leukemia complicating Hodgkin's disease: a review. Cancer 1980; 46: 1247-I 252.

3. Schimpff S., Serpick A., Stoler B. et al.: Varicellazoster infection in patients with cancer. Ann Intern Med 1972; 76: 241-254.

4. Reboul F., Donaldson S.S., Kaplan H.S.: Herpes zoster and varicella infections in children with Hodgkin's disease. Can cer 1978; 41: 95-99.

5. Lange B., Littman P.: Management of Hodgkin's disease in children and adolescents. Cancer 1983; 51: 1371.1377 .

6. Rowland K.M., Murthy A.: Hodgkin's disease: Long-term effects of therapy. Med Pediat Oncol $1986 ; 14: 88-96$.
7. Goffinet D.R., Glatstein E.J., Merigan T.C.; Herpes zosterararicella infections and lymphoma. Ann Intern Med 1972; 76: 235-240.

8. Bodey G.P.: In fectious complications in the cancer patient. CPCa 1977; 1 (12): 1-63.

9. Feldman S., Hughes W.T., Daniel C.B.; Varicella in children with cancer: Seventy-seven cases. Pediatrics $1975 ; 56: 388-397$.

10. Feldman S., Hughes W.T., Kim $H . Y$.: Herpes zoster in children with cancer. Am J Dis Child 1973; 126: 178-184.

11. Gershon A.A., Steinberg S.P., Gelb L.: Live attenuated varicella vaccine use in immunocom promised children and adults. Pediatrics $1986 ; 78$ : 757.762 .

12. Balfour H.A., Groth K.E., Mc Millough J. et al.: Prevention or modification of varicella using zoster immune plasma. Am J Dis Child 1977; 131 : 693696.

13. Shulman S.T.: Acyclovir treatment of disseminated varicella in childhood malignant neoplasms. AJDC 1985; 139: 137-140.

14. Whitley $R$., Hitty M., Haynes $R$. et al.: Vidarabine therapy of varicella in immunosuppressed patients. J Ped atr 1982; 101: 125.131.

15. Zolezzi P., Rubio J., Cardemit B. et al: Enfermedad de Hodgkin en el nin̆o. Tratamiento combinado COPP modíficado y radioterapia en estadios avanzados. Rev Chil Pedjatr 1990; 61: 74-77.

16. Miliouskas J.R., Webber B.L.: Disseminated varicella at autopsy in children with cancer. Cancer 1984; 53 : 1518-1525.

17. Pizzo P.A.: Infectious complications in the child with cancer: Il. Management of specifjc infectious organisms. I Pediatr 1981; 98: 513-523.

18. Report of the Committee on Infectious Diseases (Red Book). American Academy of Pediatrics, Illinois, 1988; 456-462.

19. Straus S.E., Ostrove J.M., Inchauspé G. et ai.: Varicella-zoster visus infections. Ann Intern Med 1988; 108: 221-237.

20. Arvin A.M., Kushner J.H., Feldman S. Baehner R.L., Hammond D., Merigan T.C.: Human leukocyte interferon for the treatment of varicella in children with cancer. N Engl J Med 1982; 306: $761-765$.

21. Feldman S, Robertson P.K., Lott L., Thornton D.: Neurotoxicity due to adenine arabinoside therapy during varicella-zoster virus infections in immunocompromised children. I Infect Dis 1986; 154: 889.883 .

22. Prober C.G., Kirk L.E., Keeney R.E.: Acyclovir therapy of chickenpox in immunosuppressed children: A collaborative study. J Pedintr 1982; 101: $622-625$.

23. Feldiman $S$, Lott $L$ : Varicella in children with cancer: Impact of antiviral therapy and prophylaxis. Pediatrics 1987; 80: 465-472. 